\title{
Pattern of dyslipidemia among adult women with polycystic ovary syndrome in Port Harcourt, Nigeria
}

\author{
Kinikanwo I. Green ${ }^{1}$, Collins Amadi ${ }^{2 *}$
}

\begin{abstract}
${ }^{1}$ Department of Obstetrics and Gynecology, ${ }^{2}$ Department of Chemical Pathology and Metabolic Medicine, University of Port Harcourt Teaching Hospital, Port Harcourt, Nigeria
\end{abstract}

Received: 30 August 2018

Accepted: 28 September 2018

*Correspondence:
Dr. Collins Amadi,
E-mail: collins338@yahoo.com

Copyright: $\odot$ the author(s), publisher and licensee Medip Academy. This is an open-access article distributed under the terms of the Creative Commons Attribution Non-Commercial License, which permits unrestricted non-commercial use, distribution, and reproduction in any medium, provided the original work is properly cited.

\section{ABSTRACT}

Background: Dyslipidemia is adjudged the most common metabolic disorder observed among women with polycystic ovary syndrome (PCOS). However, there is paucity of data to support this hypothesis in our region. Therefore, this study was structured to evaluate the pattern of dyslipidemia among women with PCOS in Port Harcourt, Nigeria.

Methods: This was a retrospective study of 226 adult women with PCOS who had visited the Department of Chemical Pathology and Metabolic Medicine of a tertiary hospital in Nigeria over a 10-year period (1st January 2008 to 31st December 2017), aimed to evaluate the pattern of dyslipidemia in these women. Records on age, total cholesterol (Tc), triglycerides (Tg), high-density lipoprotein (HDL-c), and low-density lipoprotein (LDL-c) were collected and analyzed using Shapiro-Wilk, descriptive, chi-square, and Fisher's exact statistics. Statistical significance was set at 0.05 .

Results: The mean age of the study population was $28.40 \pm 5.75$ and ranged from $18-42$ years. Most of the study population $(55.3 \%)$ were within the age group of $20-30$ years. The most common lipid abnormalities observed among the study population were increased triglycerides $(75.7 \%)$ and decreased HDL-c (61.1\%) concentrations while the least was increased total cholesterol (17.7\%) and LDL-c (14.5\%). Dyslipidemia was observed in $80.1 \%$ of the study population and more frequent $(51.9 \%)$ within the age group of 20-30 years.

Conclusions: Dyslipidemia is common among young adult women with PCOS. Regular assessment for dyslipidemia should be mandatory in PCOS to mitigate the long-term consequences of its complications.

Keywords: Dyslipidemia, Nigeria, Polycystic ovary syndrome, PCOS

\section{INTRODUCTION}

Polycystic ovary syndrome (PCOS) is a long-term recognized complex and heterogeneous disorder that affects reproductive-aged women and presents with varying degrees of gynecological, reproductive, endocrine, metabolic, and various organ-specific aberrations. ${ }^{1,2}$ The disorder is characterized by chronic ovulatory dysfunction, hyperandrogenism, and polycystic ovaries on ultrasound. ${ }^{2}$ It was in 1935 that Irving F. Stein and Michael L. Leventhal first described the clinical, macroscopic, and histological characteristics of the disorder and its association with hirsutism and amenorrhea. ${ }^{3}$ Its reported global prevalence among reproductive-aged women is about $10 \%$, however, the prevalence varies with races and ethnicities. ${ }^{4} \mathrm{~A}$ recent study had reported a $12.2 \%$ prevalence of the disorder among Nigerian women with the syndrome. ${ }^{5}$ The syndrome is common in Nigeria occurring in approximately one in six infertile Nigerian women. ${ }^{6}$

The etiopathogenesis of PCOS is ill-defined and very obscure. ${ }^{7}$ However, several theories had been suggested as possible favored factors in the evolution of the disorder. ${ }^{8}$ Currently, the interaction of certain genes with various environmental factors is the most accepted 
pathophysiologic event in the evolution of the syndrome. ${ }^{8,9}$ Due to the varied clinical features of the syndrome, it is usually defined and phenotyped based on either the 1990 National Institute of Health, the 2006 Androgen Excess Society, or the 2003 Rotterdam International Consensus criteria. ${ }^{10-12}$ Over the decades since it was first described in the literature, numerous evidence from various epidemiological reports had noted that the disorder is associated with distinct metabolic aberrations including obesity, insulin resistance, type 2 diabetes mellitus, hyperinsulinemia, hyperandrogenism, and dyslipidemia. ${ }^{13}$

Dyslipidemia is the most persistent and prevalent metabolic aberration observed among women with the syndrome. ${ }^{14}$ These lipid aberrations had been ascribed to various metabolic perturbances such as obesity, insulin resistance, and hyperandrogenemia associated with the syndrome which enhances the risk of adverse cardiovascular events in women with the syndrome in various studies. ${ }^{15}$ However, many of these studies are all confined to western populations with paucity of data in our region. Therefore, this study was designed to investigate the pattern of dyslipidemia among adult reproductive-aged women with PCOS in Port Harcourt, Nigeria. The specific objectives of the present study were to determine the prevalence of dyslipidemia among the study population, to determine the variants of dyslipidemia among the study population, to determine the pattern of dyslipidemia in various age groups of the study population, and to compare the findings from this study with similar reports around the world.

\section{METHODS}

\section{Study Area and location}

This present study was carried out in the Department of Chemical Pathology and Metabolic Medicine of the University of Port Harcourt Teaching Hospital (UPTH), Port Harcourt, Nigeria. UPTH is one of the tertiary care hospitals situated in the Niger Delta region of Nigeria. The hospital serves as a referral center for all the primary and secondary health centers in the region and the neighboring states. The Department of Chemical Pathology and Metabolic Medicine of the hospital carries out routine and complex clinical chemistry analysis and also has an attached Metabolic Clinic where patients with uncomplicated metabolic disorders, including PCOS, are referred to from different points within the hospital.

\section{Study design and structure}

The study is a retrospective, descriptive, and crosssectional study of laboratory variables including age, plasma total cholesterol (Tc), plasma high density cholesterol (HDL-c), plasma low density cholesterol (LDL-c), and plasma triglycerides (Tg) of all patients with diagnosed PCOS irrespective of phenotype who presented for fasting plasma lipid profile in the
Department of Chemical Pathology and Metabolic Medicine of the University of Port Harcourt Teaching hospital (UPTH) from different points within the hospital between 1st January, 2008 to 31st December, 2017. All the patients with PCOS had been diagnosed by the specialist gynecologist in UPTH based on the Rotterdam International consensus criteria which is defined as the presence of any two of the following three features of the syndrome $^{12}$;

- Chronic oligo-ovulation/anovulation evidenced biochemically by a mid-luteal phase (day 21-23) menstrual cycle serum progesterone of less $10 \mathrm{ng} / \mathrm{ml}$ $(32 \mathrm{nmol} / \mathrm{l})$ or clinically by oligomenorrhea/amenorrhea.

- Hyperandrogenism evidenced either clinically (hirsutism, acne, and androgenic alopecia) or biochemically (serum total testosterone level of greater than $2.8 \mathrm{nmol} / \mathrm{l}$ )

- Polycystic ovaries on transvaginal ultrasound scan (presence of twelve or more follicles measuring 2$9 \mathrm{~mm}$ in diameter and/or at least one enlarged ovary measuring more than $10 \mathrm{~cm}^{3}$ ).

\section{Inclusion criteria}

- Records of fasting lipid profile of patients more than 18 years of age with any phenotype of PCOS.

\section{Exclusion criteria}

- Records of fasting lipid profile of PCOS patients who are either pregnant, on lipid-lowering medications, with established diabetes mellitus, with androgen excess disorders, hyperprolactinemia, thyroid disorders including those with incomplete data.

\section{Specimen collection, processing, and laboratory analysis}

During the period under study, fasting venous whole blood specimen were collected from each patient by phlebotomy through the antecubital vein while adhering to the basic universal safety precautions. All collected specimen were emptied into ethylenediaminetetracetic acid (EDTA) specimen collection tubes and subsequently processed accordingly.

Laboratory analysis for plasma Tc, HDL-c, and and Tg were all carried out through the enzyme-catalyzed colorimetric methods with same brands of laboratory reagents including three levels (levels 1, 2, and 3) of commercial quality control sera sourced from Randox Laboratories, United Kingdom.

While the plasma LDL-c were calculated using the following Friedewald formula. ${ }^{16}$

$\mathrm{Tc}-\mathrm{HDL}-\mathrm{c}-\mathrm{Tg} / 2.2$ 
All parameters in the Friedewald formula were all in mmol/l unit.

The Friedewald formula was only applied if fasting plasma triglyceride (Tg) level was less than $4.5 \mathrm{mmol} / \mathrm{l}$.

\section{Data Collection, stratification, and definitions}

All records from laboratory result sheets and case notes of each PCOS patient were collected, reviewed and entered into Statistical Package for Social Sciences (SPSS) version 20. Records collected were demographic data (age), clinical diagnosis (PCOS), total cholesterol (Tc) in mmol/l, high-density cholesterol (HDL-c) in mmol/l, triglycerides ( $\mathrm{Tg}$ ) in $\mathrm{mmol} / \mathrm{l}$ and the calculated low-density lipoprotein (LDL-c) in mmol/l.

Age of study cohorts was arbitrarily stratified in four groups as follows: < 20 years, 20 - 30 years, $30-40$ years, and $>40$ years.

Dyslipidemia was defined in this study based on the National Cholesterol Education Program-Adult Treatment Panel 111 (NCEP-ATP 111) as the presence of any of the following plasma lipid profile ${ }^{17}$;

- Total cholesterol of greater than $5.17 \mathrm{mmol} / \mathrm{l}$

- High-density cholesterol less than $1.3 \mathrm{mmol} / \mathrm{l}$

- Triglycerides greater than $1.7 \mathrm{mmol} / \mathrm{l}$

- Low-density lipoprotein greater than $3.36 \mathrm{mmol} / \mathrm{l}$

Therefore, based on the NCEP-ATP 111 criteria, Tc was stratified as normal $(<5.17 \mathrm{mmol} / \mathrm{l})$ or abnormal $(>5.17 \mathrm{mmol} / \mathrm{l})$, HDL-c stratified as normal (> 1.3 mmol/l) or abnormal $(<1.3 \mathrm{mmol} / \mathrm{l}), \mathrm{Tg}$ stratified as normal $(1.7 \mathrm{mmol} / \mathrm{l})$ or abnormal $(>1.7 \mathrm{mmol} / \mathrm{l})$, and the calculated LDL-c stratified as normal $(<3.36 \mathrm{mmol} / \mathrm{l})$ or abnormal (> $3.36 \mathrm{mmol} / \mathrm{l})$

\section{Statistical analysis}

All the acquired data were entered into the SPSS version 20 software. Subsequently, the data were all reviewed, coded, and properly validated. The non-Gaussian distributed data were logarithmically transformed prior to statistical analysis. Analysis was done using ShapiroWilk test, descriptive statistics, Chi-square test, and Fisher's exact test. Probability value of less than 0.05 were regarded as statistically significant.

\section{RESULTS}

During the 10-year period (1st January 2008 to December 2017), two hundred and thirty-four (234) patients with PCOS presented to the Department of Chemical Pathology and Metabolic Medicine for fasting lipid profile investigations using fasting plasma Tc, HDL-c, and $\mathrm{Tg}$ lipid panels. Among the plasma lipid panels of these 234 patients who presented during that period under study, the data of 226 (which is $96.6 \%$ of the total) of these patients met the inclusion criteria of the present study and were recruited for the present study.

Table 1: Characteristics of study variables.

\begin{tabular}{|c|c|c|c|}
\hline Variables & $\mathbf{n}$ & Range & Mean $\pm 2 S D$ \\
\hline Age (years) & 226 & $18-42$ & $28.40 \pm 5.75$ \\
\hline Plasma Tc (mmol/l) & 226 & $3.0-5.7$ & $4.34 \pm 0.65$ \\
\hline Plasma HDL-c mmol/l) & 226 & $0.5-1.7$ & $1.10 \pm 0.34$ \\
\hline Plasma Tg (mmo/l) & 226 & $1.5-6.7$ & $3.40 \pm 1.58$ \\
\hline Plasma LDL-c (mmol/l) & 166 & $1.1-4.8$ & $1.90 \pm 0.65$ \\
\hline $\begin{array}{l}\mathrm{D}=\text { Standard deviation, } \\
\text { lensity lipoprotein cholester } \\
\text { lensity lipoprotein cholester }\end{array}$ & $\mathrm{Tc}=\mathrm{T}$ & inorid & HDL-c $=\mathrm{H}$ \\
\hline
\end{tabular}

Records of eight (8) of these PCOS patients did not meet the study inclusion criteria and were subsequently excluded.

Shapiro-Wilk test was initially used to determine the degree of skewness of the study variables before further statistical analysis. Following the Shapiro-Wilk statistical analysis of study variables, plasma HDL-c (Z-score = +21.446 ; $\mathrm{p}$-value $=0.001)$ and LDL-c $(Z$-score $=+4.142$; $\mathrm{p}$-value $=0.002$ ) were found to be skewed while age, Tc, and $\mathrm{Tg}$ were all normally distributed. Subsequently, the HDL-c and LDL-c data were logarithmically transformed to maintain these variables in Gaussian distribution (nonskewed) prior to analysis.

In table 1 , the age of the study population ranged from 18 to 42 years with a mean of $28.40 \pm 5.57$ years. The range and mean \pm standard deviation of plasma Tc, HDL-c, Tg, and the calculated LDL-c are also shown.

Table 2: Age distribution of the study population.

\begin{tabular}{|c|c|c|c|c|}
\hline $\begin{array}{l}\text { Age groups } \\
\text { (years) }\end{array}$ & $\mathbf{N}$ & $\%$ & Fisher's Exact & p-value \\
\hline$<20$ & 19 & 8.4 & \multirow{4}{*}{164.903} & \multirow{4}{*}{$<0.001 *$} \\
\hline $20-30$ & 125 & 55.3 & & \\
\hline $30-40$ & 78 & 34.5 & & \\
\hline$>40$ & 4 & 1.8 & & \\
\hline
\end{tabular}

*Statistically significant

The mean of the plasma HDL-c concentration among the study population was decreased as adjudged by NCEPATP 111 definition. The mean plasma level of the Tg was also high among the study population based on the NCEP-ATP 111 definition.

Table 3: Prevalence of dyslipidemia among study population based on the national cholesterol education program-adult treatment panel 111 criteria.

\begin{tabular}{|llll|}
\hline \multirow{2}{*}{ Dyslipidemia } & Negative & 45 & $\%$ \\
\cline { 2 - 3 } & Positive & 181 & 80.1 \\
\hline \multicolumn{2}{|l|}{ Chi-square $=13.897 ;$ p-value $=<0.001$} & \\
\hline
\end{tabular}


The plasma level of $\mathrm{Tg}$ of sixty $(n=60)$ of the study population was more than $4.5 \mathrm{mmol} / \mathrm{l}$, hence only those ( $\mathrm{n}=166$ ) with plasma $\mathrm{Tg}$ level less than $4.5 \mathrm{mmol} / \mathrm{l}$ had their LDL-c calculated using the Friedewald formula in this study. In table 2, the majority of the study population are within the age group 20-30 years $(55.3 \%)$ with the least study population $(1.8 \%)$ within the age group of more than forty years. In Table 3 , majority $(80.1 \%$; $\mathrm{n}=$
181) of the study population had defined dyslipidemia based on the NCEP-ATP 111 criteria.

In Table 4, based on the NCEP-ATP 111 plasma lipid limits, there were significant difference between those with normal and abnormal plasma lipid levels of Tc, HDL-c, Tg, and LDL-c.

Table 4: Stratification of plasma lipid levels of study population based on the national cholesterol education program-adult treatment panel 111 limits.

\begin{tabular}{|lllcl|}
\hline Lipid variables & Based on NCEP-A TP 111 Criteria & & \\
& Normal n $(\%)$ & Abnormal n $(\%)$ & Chi-square & P-value \\
\hline Plasma Tc (mmol/l) & $186(82.3)$ & $40(17.7)$ & 94.319 & $<0.001^{*}$ \\
\hline Plasma HDL-c (mmol/l) & $88(38.9)$ & $138(61.1)$ & 10.195 & $0.001^{*}$ \\
\hline Plasma Tg (mmol/l) & $55(24.3)$ & $171(75.6)$ & 15.735 & $<0.001^{*}$ \\
\hline Plasma LDL-c (mmol/l) & $142(85.5)$ & $24(14.5)$ & 97.097 & $<0.001^{*}$ \\
\hline
\end{tabular}

*Statistically Significant; NCEP-ATP 111 = National Cholesterol Education Program-Adult Treatment Panel 111; Tc = Triglycerides; HDL-c = High Density Lipoprotein Cholesterol; Tg = Triglycerides; LDL-c = Low Density Lipoprotein Cholesterol.

However, the most common lipid abnormality is elevated $\mathrm{Tg}(75.6 \%)$ followed by decreased HDL-c (61.1\%). While the least lipid abnormality was elevated Tc $(17.7 \%)$ and elevated LDL-c (14.5\%). In Table 5, dyslipidemia was observed more $(51.9 \%)$ within the study population of age group 20-30 years.

Table 5: Distribution of dyslipidemia based on each age group of the study population.

\begin{tabular}{|lllll|}
\hline & \multicolumn{4}{c}{ Age groups (years) } \\
$\begin{array}{l}\text { Status of } \\
\text { Dyslipidemia }\end{array}$ & $<20$ & $20-30$ & $30-40$ & $>40$ \\
$\begin{array}{l}\text { Negative n } \\
(\%)\end{array}$ & 9 & 31 & 5 & $0(0 \%)$ \\
\hline $\begin{array}{l}\text { Positive n } \\
(\%)\end{array}$ & 10 & 94 & 73 & 4 \\
\hline
\end{tabular}

Fisher Exact; $\mathrm{p}$-value $=<0.001$

\section{DISCUSSION}

PCOS had historically been characterized as a disorder of the female reproductive function and that of fertility. ${ }^{1}$ This assumption stem from the report in 1935 by Stein and Leventhal who had described the syndrome as a triad of hirsutism, amenorrhea, and polycystic ovaries. ${ }^{3}$ However, with the decade-long intense research on its varied clinical features, etiology, pathophysiology coupled with the improved diagnostic capabilities of the disorder, the syndrome has gained prominent attention as a rather multi-complex disorder with various gynecological, reproductive, endocrine, and metabolic consequences. ${ }^{8,9}$ It has been linked to the increased risk of obesity, insulin resistance, impaired glucose tolerance, type 2 diabetes mellitus, metabolic syndrome, nonalcoholic fatty liver disease, hypertension, and dyslipidemia. ${ }^{13}$ However, the most frequent metabolic aberration observed among women with the syndrome in recent time is dyslipidemia. ${ }^{18}$

In this present study, we had evaluated the pattern of lipid and lipoprotein pattern among adult PCOS patients based on the NCEP-ATP 111 criteria and observed $80.1 \%$ incidence of dyslipidemia which is in agreement with the $80 \%$ incidence of dyslipidemia reported recently from Pakistan. ${ }^{19}$ Moreover, the high incidence of dyslipidemia obtained in this study is almost in accord with the $76.7 \%$ incidence of dyslipidemia observed among patients with PCOS using the NCEP-ATP 111 criteria in a similar study. ${ }^{20}$ However, some other authors had reported lower frequencies of dyslipidemia among women with PCOS. ${ }^{21-}$ 22 It has been reported that young adult women with PCOS are more at risk of dyslipidemia than their older counterparts due to the increased incidence of obesity, insulin resistance, and the androgen excess state inherent in these young adult women with PCOS. ${ }^{23-24}$ These reports are all in accord with this study where dyslipidemia was found to be more frequent $(55.3 \%)$ among the young adult women within the age group of 20-30 years.

Although multiple studies have demonstrated an increased incidence of dyslipidemia among women with PCOS in recent time, however, the specific pattern of these lipid disorders varies among these studies. ${ }^{25-27}$ Some studies had reported an increased level of LDL-c as the most lipid abnormality, some had documented only low HDL-c cholesterol as the only abnormality observed in women while others have reported elevated Tc as the only abnormality. ${ }^{25}$ However, the most consistent lipid abnormality reported in most studies is the increased $\mathrm{Tg}$ level associated with low HDL-c level. ${ }^{26-27}$ The finding in 
this study is in agreement with most of these studies in support of the high Tg and low HDL-c levels among the majority of women with PCOS. Using the NCEP-ATP 11 criteria in this study, most $(75.7 \%)$ of the study population had elevated $\mathrm{Tg}$ levels while $61.1 \%$ had low HDL-c levels. The conflicting variations of the pattern of dyslipidemia among these studies could be related to genetic and environmental factors which influences plasma lipid levels in women with PCOS. ${ }^{28}$

Reproductive-aged women with PCOS harbor clusters of metabolic factors such as obesity, insulin resistance and hyperandrogenism which all act in concert to increase the risk of these adverse cardiovascular events in this group of women compared with the general population. ${ }^{29}$ Cardinal to the development of these adverse cardiovascular events is the formation of atherosclerosis which is ultimately enhanced among these women in presence of dyslipidemia. ${ }^{29-30}$ Patients with PCOS have a higher prevalence of atherosclerosis and an estimated sevenfold increased likelihood of myocardial infarction which are pathological events linked to dyslipidemia. ${ }^{31}$ This factor underscores the expert-suggested need for regular assessment for dyslipidemia among these patients with PCOS. ${ }^{32}$ The pathogenesis of dyslipidemia in PCOS has been ascribed to various metabolic consequences of the syndrome. However, there is some form of uniformity of its involvement with insulin resistance and that of hyperandrogenism among most researchers. ${ }^{13-16}$

\section{CONCLUSION}

The findings of this study are suggestive of high incidence of dyslipidemia among women with PCOS, especially the young adult women. Therefore, regular assessment for dyslipidemia among patients with PCOS should be mandatory as part of their management modalities to mitigate the long-term consequences of dyslipidemia.

\section{ACKNOWLEDGMENTS}

Authors would like to thank all the laboratory staff for their assistance and technical support during the study.

Funding: No funding sources

Conflict of interest: None declared

Ethical approval: The study was approved by the Institutional Ethics Committee

\section{REFERENCES}

1. Alsadi B. Polycystic Ovarian Syndrome: Pathophysiology and Infertility. World J Lap Surg. 2014;7(1):23-27.

2. Moharaj S, Amod A. Polycystic ovary syndrome. J Endocrinol Metab S Afr. 2009;14(2): 86-95.

3. Stein IF, Leventhal ML. Amenorrhoea associated with bilateral polycystic ovaries. Am J Obstet Gynecol. 1935;29:181-91.
4. Lavie O. Benign disorders of the ovaries and oviducts. In: Alan HD, Lauren N, Ashley SR, editors. Current Obstetrics and Gynaecologic Diagnosis and Treatment. $11^{\text {th }}$ ed. New York: Lange Medical Publication; 2013. pp. 661-70.

5. Omokanye LO, Ibiwoye-Jaiyeola OA, Olatiowo AWO, Abdul IF, Durowade KA, et al. Polycystic ovary syndrome. Analysis of management outcomes among women at a public health institution in Nigeria. Niger J Gen Pract. 2015;13(2):44-8.

6. Ugwu GO, Iyoke CA, Onah HE, Mba SG. Prevalence, presentation and management of polycystic ovary syndrome in Enugu, South-east Nigeria. Niger J Med. 2013;22(4):313-6.

7. Azziz R, Carmina E, Chen Z, Dunaif A, Laven JS, Legro RS et al: Polycystic ovary syndrome. Nat Rev Dis Primers. 2016;2:16057.

8. Goodarz MO, Dumesic DA, Chazenbalk G, Azziz R. Polycystic ovary syndrome: etiology, pathogenesis and diagnosis. Nat Rev Endocrinol. 2011;7(4):21931.

9. Joe-Kechebelu NN, Mbamara SU, Ikechebelu JI. Familial trend in polycystic ovarian syndrome. Ann Afr Med. 2013;12(3):132-4.

10. Zawadic JK, Danif A. Diagnostic criteria for polycystic ovarian syndrome: towards a rational approach. In: Polycystic Ovary Syndrome, Dunaif A, Givens JR, Haseltine F (Eds.). Cambridge, England: Blackwell Science, 1992. pp. 377-84.

11. Azziz R, Carmina E, Dewailly D, DiamantiKandarakis E, Escobar-Morreale HF, Futterweit W, et al. Positions statement: criteria for defining polycystic ovary syndrome as a predominantly hyperandrogenic syndrome: an Androgen Excess Society guideline. J Clin Endocrinol Metab. 2006; 91(11):4237-45.

12. Rotterdam, ESHRE/ASRM-Sponsored, PCOS, Consensus, Workshop, Group. Revised 2003 consensus on diagnostic criteria and long-term health risks related to polycystic ovary syndrome. Fertil Steril. 2004; 81(1):19-25.

13. Churchill SJ, Wang ET, Pisarska MD. Metabolic consequences of polycystic ovary syndrome. Minerva Ginecol. 2015;67(5):545-55.

14. Berneis K, Rizzo M, Hersberger M, Rini GB, Di Fede G, Pepe I, et al. Atherogenic forms of dyslipidemia in women with polycystic ovary syndrome. Int J Clin Pract. 2009;63(1):56-63.

15. Wild RA, Rizzo M, Clifton S, Carmina E. Lipid levels in polycystic ovary syndrome: systematic review and meta-analysis. Fertil Steril. 2011;95(3): 1073-9.

16. Friedewald WT, Levy RI, Fredrickson DS. Estimation of the concentration of low-density lipoprotein cholesterol in plasma, without the use of the preparative ultracentrifuge. Clin Chem. 1972; 18(6):499-502.

17. Executive summary of the third report of the National Cholesterol Education Program (NCEP) Expert Panel on Detection, Evaluation and Treatment 
of High Blood Cholesterol in Adults (Adult Treatment Panel III). JAMA 2001;285(19):2486-97.

18. Legro RS, Kunselman AR, Dunaif A. Prevalence and predictors of dyslipidemia in women with polycystic ovary syndrome. AM J Med. 2001;111(8):607-13.

19. Hussain A, Alam JM. Dyslipidemia in women with polycystic ovary syndrome: A case control study in tertiary care hospital of Karachi. J Pak Med Asso. 2014;64(90):1049-52.

20. Rocha MP, Marcondes JA, Barcellos CR. Dyslipidemia in women with polycystic ovary syndrome: incidence, pattern and predictors. Gynecol Endocrinol. 2011;27(10):814-9.

21. Nisa SUN, Nisa SSUN, Nasreen S. Frequency of dyslipidemia in patients of polycystic ovarian syndrome (PCOS) in BV hospital, Bahawalpur. Pak J Med Health Sci. 2014;8(2):489-91.

22. Kim JJ, Choi YM. Dyslipidemia in women with polycystic ovary syndrome. Obs Gynecol Sci. 2013; 56(3):137-42.

23. Wild RA, Painter PC, Coulson PB, Carruth KB, Ranny GB. Lipoprotein lipid concentrations and cardiovascular risk in women with polycystic ovary syndrome. J Clin Endocrinol Metab. 1985;61(5): 946-51.

24. Carmina E. Cardiovascular risk and events in polycystic ovary syndrome. Climacteric. 2009;12 (suppl 1):22-5.

25. Shoaib OM, Mustafa SM, Nourein IH. Serum lipid profile of polycystic ovary syndrome in Sudanese women. Int J Med Sci Public health. 2015;4(11): 1605-10.

26. Robinson S, Henderson AD, Gelding SV, Kiddy D, Niththyananthan R, Bush A, et al. Dyslipidaemia is associated with insulin resistance in women with polycystic ovaries. Clin Endocrinol (Oxf). 1996; 44(3):277-84.

27. Meirow D, Raz I, Yossepowitch O, Brzezinski A, Rosler A, Schenker JG, et al. Dyslipidaemia in polycystic ovarian syndrome: different groups, different aetiologies? Hum Reprod. 1996;11(9):1848-53.

28. Essah PA, Nestler JE, Carmina E. Differences in dyslipidemia between American and Italian women with polycystic ovary syndrome. J Endocrinol Invest. 2008;31(1):35-41.

29. Guzick DS1, Talbott EO, Sutton-Tyrrell K, Herzog HC, Kuller LH, Wolfson SK Jr. Carotid atherosclerosis in women with polycystic ovary syndrome: initial results from a case-control study. Am J Obstet Gynecol. 1996;174(4):1224-9.

30. Dahlgren E1, Janson PO, Johansson S, Lapidus L, Oden A. Ovary syndrome and risk for myocardial infarction. Evaluated from a risk factor model based on a prospective population study. Acta Obstet Gynecol Scand. 1992;71(8):559-604.

31. Wild RA; Long-term health consequences of PCOS. Hum Reprod Update. 2002;8(3):231-41

32. American College of Obstetricians and Gynecologist committee on practice bulletins - Gynecology. ACOG practice bulletin no. 108: polycystic ovary syndrome. Obstet Gynecol. 2009;114:936-49.

Cite this article as: Green KI, Amadi C. Pattern of dyslipidemia among adult women with polycystic ovary syndrome in Port Harcourt, Nigeria. Int J Reprod Contracept Obstet Gynecol 2018;7:4493-8. 